\title{
Eclampsia in Janakpur Zonal Hospital, Nepal: Favourable outcome with Magnesium sulphate
}

\author{
Rani Jha, Sheela Verma, Shailesh K Jha \\ Dept of Ob/Gyn Janakpur Zonal Hospital, Nepal
}

\begin{abstract}
Aim: To determine the demographic characters of patients of eclampsia and the effectiveness of Magnesium Sulphate in terms of maternal and perinatal outcome.

Methods: A prospective study of the eclamptic patients was conducted from 18 th Aug - $17^{\text {th }}$ Oct 2006 (1 Bhadra 2063 to 30 Ashwin 2063) attending the Obstetric Department of Janakpur Zonal Hospital (JZH).

Results: Of the 50 eclamptic women [antepartum 80\%, intrapartum $6 \%$ and postpartum 14\%] with overall improvement in $92 \%$ although 2 cases had to be referred to center with ICU facility and 2 cases died. The demographic characteristics were young primiparous population of low socioeconomic condtion. Caesarean was done for $48 \%$. Pretermaurity was seen in 50\% and $76 \%$ were live birth.

Conclusions: Nepal has a high maternal mortality rate and eclampsia is a big contributing factor, which could be reduced by using magnesium sulphate widely in all parts of Nepal taking, ours as one of the peripheral hospital as an example.
\end{abstract}

Key words: Eclampsia, magnesium sulphate, maternal mortality

\section{Introduction}

Large randomized trials in developing countries and systematic reviews have shown the usefulness of magnesium sulphate in treating recurrent eclamptic seizures and in the prophylaxis of eclampsia ${ }^{1,2}$. Despite this evidence magnesium sulphate remains underused.

A large number of eclamptic patients with consistent rise as winter approaches attend the Obstetric Department of Janakpur Zonal Hospital (JZH). Magnesium sulphate is used for the treatment of all patients of eclampsia was found to have better prognosis. Besides this, mode of delivery has been influenced by control of fits and fetal maturity. Hence we set forth to determine the demographic characters of eclamptic women and also to dissipate the effectiveness of magnesium sulphate, in terms of maternal and perinatal outcome in Terai population of Nepal.

\section{Methods}

A prospective study of eclamptic patients was conducted from $18^{\text {th }}$ Aug $-17^{\text {th }}$ Oct 2006 (1 Bhadra 2063 to 30 Ashwin 2063) All the patients of eclampsia admitted during this period in JZH were included in the study. Findings at the time of admission were noted. Patients were followed-up everyday till the time of discharge. A prepared and pre-tested questionnaire was administered to all the patients after recovery. If patient was unable to answer the queries one of the closest relative, staying in the same household was asked the questions. Modality of management, maternal and perinatal outcome was noted.

\section{Corresspondence}

Rani Jha, MD, Consultant Obstetrician and Gynecologist, JZH

Sheela Verma, MD, Senior Consultant Obstetrician and Gynecologist, Unit in-charge, Obs-Gyn, JZH

SK Jha, MDGP, Asst. Professor, Dept.of Medicine (full form plz)

Janaki Medical College Teaching Hospital, Janakpur

Email: ranijha555@hotmail.com 


\section{Results}

Total no. of eclamptic patients were 50 . Out of these 50 eclamptics, $46 \%$ were below 18 years of age at the time of pregnancy. Additional $40 \%$ were of 19-22 years of age. Those belonging to poor socio-economic class were $32 \%$ (fig1). Illiteracy was seen in $28(56 \%)$.

Antepartum eclampsia was the most important finding making $80 \%$ of the total. This was followed by intrapartum and postpartum eclampsia $6 \%$ and $14 \%$ respectively. Of all these women $88 \%$ were nullipara in comparison to $12 \%$ multiparas. Gestational age was less than 37 weeks among 50\% of the women at the time of delivery. $10 \%$ women were not sure of gestational age and scan could not be performed in these cases to confirm the gestational age. At admission, Systolic BP was below $160 \mathrm{~mm}$ of $\mathrm{Hg}$ in $82 \%$ women, whereas diastolic BP was below $110 \mathrm{~mm}$ of $\mathrm{Hg}$ in $90 \%$ of them (Table 1and 2).

All these cases were resuscitated and one of the cases upon arrival could not be revived as she was already gasping. Stabilization was tried and one died while such effort was on process.

Two were stabilized and referred to Teaching Institute, while the rest of them were managed in our own hospital. Pritchard Regime of magnesium sulphate was followed. There were recurrence of fits but these were in few women. For control of hypertension, the drugs preferably used were Nefedepine.

Spontaneous vaginal delivery occurred in $42 \%$ of all the deliveries in the hospital with additional $14 \%$ occurring at home forming total vaginal delivery to be $62 \%$ including all other types of vaginal deliveries (6\%). Rests $48 \%$ were delivered by LSCS done for eclampsia. Of all the deliveries $76 \%$ live birth. In terms of maternal benefit $92 \%$ women had their fits controlled by Magnesium sulphate.

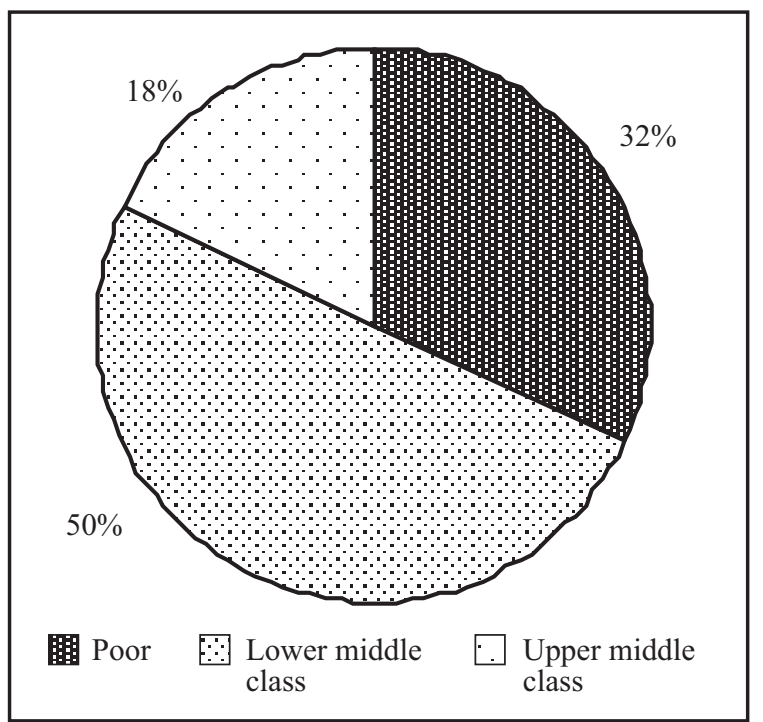

Table I. Blood pressure at admission

\begin{tabular}{|l|c|c|}
\hline Systolic BP & No. & $\%$ \\
\hline$<140$ & 23 & 46 \\
$141-150$ & 5 & 10 \\
$151-160$ & 13 & 26 \\
$>160$ & 9 & 18 \\
\hline
\end{tabular}

Table 2. Blood pressure at admission

\begin{tabular}{|l|c|c|}
\hline Diastolic BP & No. & $\%$ \\
\hline$<90$ & 14 & 28 \\
$91-100$ & 22 & 44 \\
$101-110$ & 9 & 18 \\
$>110$ & 5 & 10 \\
\hline
\end{tabular}

Two women were referred to other hospital for ICU management and unfortunately other two women died during the course of treatment.

\section{Discussion}

It is surprising to find such a huge number of eclamptic women in the span of such a short time. Even 10 years data of a hospital with 3200 deliveries had 18 cases of eclampsia and 297 severe preeclampsia. ${ }^{3}$ The demographic aspects, showing early age at child bearing to be a contributing factor rather than pregnancy at older age in eclampsia. ${ }^{4}$ As is shown by the studies of Conde-Aguedlo ${ }^{5}$ nullipara comes up as a significant contributory factor in our study. As expected, incidence of eclampsia is not far less among socioeconomically advantageous group of women. ${ }^{6}$

Magnesium sulphate has already been established as an effective management for control of convulsion in eclampsia, but is still being underused. ${ }^{1,2,7}$ The study conducted in $\mathrm{JZH}$ reinforces the effectiveness of Magnesium sulphate by showing maternal improvement $92 \%$.

However intubation facility for women in gasping condition upon arrival would have a proven benefit as ICU management imperative. This could give life to already moribund women, even in association of HELLP syndrome and renal failure. ${ }^{8}$

Eclampsia being seen in such a huge number, prophylaxis against preeclmapsia is worth taking a consideration. For this at least pregnant women should have antenatal care coverage. It is rather wise to prevent worsening of preeclampsia/severe preeclampsia to eclampsia rather than to treat the condition. 
Serum markers for predicting pre-eclampsia are placental protein 13 (PP-13), as well as soluble fms-like tyrosine kinase-1 (sFlt-1), placental growth factors (PIGF) and soluble endoglin (sEng) at a relatively early stage have shown high predictive values and improved diagnostic performance if combined with first trimester Doppler sonography. ${ }^{9}$

One third of women with raised antiphospholipid are seen to develop preeclampsia or severe preeclampsia/ eclampsia with HELLP (hemolysis, elevated liver enzymes, and low platelets). ${ }^{10}$ But there are no facilities to adhere to such modern development.

What we can do most is to popularize community teachings of symptoms of preeclampsia and severe preeclampsia. Or have a group of people know about this condition while they are on queue, waiting for their turns in antenatal OPD. The problems with preeclampsia unlike anemia, is that, this cannot be detected on general physical examination.

For this it necessary to take blood pressure measurement, this needs training. Most of the time Blood pressure readings are fallacious because of the instrument itself or because of someone who takes them.

That is why urine examination is very important. It has been found out many times, women to collect the urine home for testing. Fresh urine is necessary for albumin testing. This can be easily taught, either by dipstick or by boiling. Though there are possibilities of urine infection in positive testing, but most of the women at least can be referred to centre where there is facility to record blood pressure. But then it would be sad to have them after admission in the tertiary care. ${ }^{11}$

If all the women underwent urine testing in the community and those with positive albuminuria are referred to the nearest hospital, a lot of women would be detected and receive treatment thereby. And they would not have to be re-exposed to another pregnancy too soon to meet the fetal losses that have occurred in $1 / 4$ of cases, facing yet additional risk of iron depletion.

\section{Conclusion}

Nepal has a high maternal mortality rate and eclampsia is a big contributing factor, which could be reduced by using magnesium sulphate widely in all parts of Nepal taking ours as an example; one of the peripheral hospital, away from the capital.

\section{Acknowledgement}

Our thanks to Miss Laxmi BK, ANM student who helped us in this study.

\section{References}

1. The Magpie Trial Collaborative Group (2002). Do women with pre-eclampsia, and their babies, benefit from magnesium Sulphate? The Magpie Trial: a randomized placebo-controlled trial. Lancet, 359, 1877-90.

2. Magpie Trial Follow-Up Study Collaborative Group. The Magpie Trial: a randomised trial comparing magnesium sulphate with placebo for pre-eclampsia. Outcome for women at 2 years. BJOG. 2007 Mar; 114(3):300-9. Epub 2006 Dec 12.

3. Sobande AA, Eskandar M, Bahar A, Abusham A. Severe pre-eclampsia and eclampsia in Abha, the south west region of Saudi Arabia. J Obstet Gynaecol. 2007 Feb; 27(2):150-4.

4. Okafor UV, Efetie ER, Onwuekwe I, Uwaezeoke TC. Cerebrovascular accident with quadriplegia following postpartum eclampsia. Acta Anaesthesiol Scand. 2006 Mar; 50(3):382-4.

5. Conde-Aguedlo A, Belizan JM. Risk factors for preeclmpsia in a large cohort of Latin American and Carribean women.Br J Obstet Gynaecol $107: 75,2000$

6. Verma S: Effect of Magnesium Sulphate in Eclampsia at Maternity Hospital, Kathmandu. March 2003, Research Study, Maternity Hospital

7. Pritchard JA, Cunningham FG, Mason RA: The Parkland Memorial Hospital protocol for treatment of eclampsia: Evaluation of 245 cases. Am J Obstet Gynecol 1984; 48:951.

8. Okafor UV, Efetie RE. Acute renal failure due to HELLP syndrome and acute renal failure in mid gestation. Int J Obstet Anesth. 2005 Jul; 14(3):2658.

9. Baumann MU, Bersinger NA, Surbek DV. Serum markers for predicting pre-eclampsia. Mol Aspects Med. 2007 Apr; 28(2):227-44. Epub 2007 Apr 27.

10. Clark EA, Silver RM, Branch DW. Do antiphospholipid antibodies cause preeclampsia and HELLP syndrome? Curr Rheumatol Rep. 2007 Jun; 9(3):219-25.

11. Choudhary P. Eclampsia : a hospital based retrospective study Kathmandu University Medical Journal. 2003; 1 (4): 237-241 